Widefield ScIENCE AND TeChNOLOGy For the SKA

SKADS CONFERENCE 2009

S.A. Torchinsky, A. van Ardenne, T. van den Brink-Havinga, A.J.J. van Es, A.J. Faulkner (eds.)

4-6 November 2009, Château de Limelette, Belgium

\title{
Progress on Analogue Front end for 2PAD
}

\author{
T.S. Ikin ${ }^{1}$, P.N. Wilkinson ${ }^{1}$, A.J. Faulkner ${ }^{2}$, M. Jones ${ }^{3}$, A. Baird ${ }^{3}$, A.K. Brown ${ }^{4}$, D. George ${ }^{4}$, G. Harris ${ }^{1}$, P.L. Kibet ${ }^{5}$ \\ M. Panahi ${ }^{4}$, D. Price ${ }^{3}$, H.C. Reader ${ }^{5}$, S. Schediwy ${ }^{3}$, P.S. van der Merwe ${ }^{5}$, K. Zarb-Adami ${ }^{3}$, and Y. Zhang ${ }^{4}$ \\ 1 Jodrell Bank Centre for Astrophysics, The University of Manchester, Jodrell Bank Observatory, Macclesfield, Cheshire, SK11 \\ 9DL, $\mathrm{UK}^{\star}$ \\ 2 Cavendish Astrophysics, Department of Physics, Cavendish Laboratory, University of Cambridge, JJ Thomson Avenue, \\ Cambridge, CB3 OHE, UK \\ 3 Oxford Astrophysics, Department of Physics, Denys Wilkinson Building, Keble Road, Oxford OX1 3RH, UK \\ ${ }^{4}$ Microwave and Communication Systems Research Group, School of Electrical and Electronic Engineering, Sackville Street \\ Building, University of Manchester, M60 1QD, UK \\ ${ }^{5}$ Department of Electrical and Electronic Engineering, University of Stellenbosch, 7600 Stellenbosch, South Africa
}

\begin{abstract}
The medium of RF signal transfer adopted for 2PAD was twisted pair differential signal cabling. While intended to demonstrate a low cost solution, the technique has brought with it several challenges, not least in terms of the losses and phase skew introduced. Some interesting engineering challenges have been faced along the way to delivering usable signals to the DSP Engineers.

Faced with an aggressive RFI environment, with strong TV and GSM broadcast signals, a small, reasonably quiet band was exploited. A review of the RFI shielding policy has been required to maintain stability in the gain stages of the analogue system. An effective cabinet RFI barrier has been successfully demonstrated. Future work through PrepSKA will explore alternative cabling solutions, such as coaxial and optical fibre, with the intention of evaluating the main contenders against the cost, power, and performance requirements for SKA.
\end{abstract}

\section{Introduction}

The 2PAD (2 Polarisation All Digital) demonstrator constructed at Jodrell Bank Observatory is the result of three years of collaboration between three UK institutions: The University of Manchester; The University of Oxford and the University of Cambridge. The purpose of building this instrument has been to study and demonstrate the feasibility of an all digital antenna array, in contrast to alternative schemes which use analogue beamforming techniques (van Ardenne 2004). The strategy adopted in the design of 2PAD was to produce a highly flexible and modular system. To this end the whole telescope has many systems and subsystems which connect and complement each other to produce a working digital beamformer. Future refinements on 2PAD will focus on reducing power, cost and complexity.

While the emphasis in this project has been on the digital electronics, considerable research work has gone into the analogue front end, and this is the subject of this paper.

\section{System Overview}

The system is described in schematic form in Fig. 1 which shows a single channel. The basic requirement for this analogue chain was to provide a clean signal with sufficient gain and bandwidth to drive the input of an 8-bit analogue to digital converter (ADC) in the digital back-end. Since the ADC

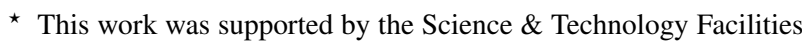
Council, and by the European Commission Framework Program 6, Project SKADS, Square Kilometre Array Design Studies (SKADS), contract no 011938 .
Table 1: Configuration of 2-PAD

\begin{tabular}{ll}
\hline Polarisations & 2 \\
Initial Array Configuration & $4 \times 4$ \\
Final Array Configuration & $8 \times 8$ \\
Scan Angle & $\pm 45^{\circ}$ \\
Bandwidth & $300 \mathrm{MHz}$ to $1000 \mathrm{MHz}$ \\
\hline
\end{tabular}

direct-samples the RF signal, there was no requirement for RF conversion.

Referring to Fig. 1 we may trace the main modules in the system. These will be described in more detail below. Firstly, the antenna signal is converted to coaxial by a balun transformer. This signal is amplified by an LNA before being bandlimited in a filter module. Band-limiting is required to reduce the intermodulation effects in subsequent stages. Four bandlimited antenna signals are treated in one Gain Chain Module. In this unit there are three gain blocks and a transformer which drives one twisted pair of the category-7 (CAT-7) cable. At the far end of the 20 meter cable the signals are converted from balanced back to single ended. Before being digitised, a Signal Conditioning Module provides various optional stages of filtering and attenuation. The Signal Conditioning Module also provides the power to the front-end electronics using the same twisted pair wires as the RF signal. 


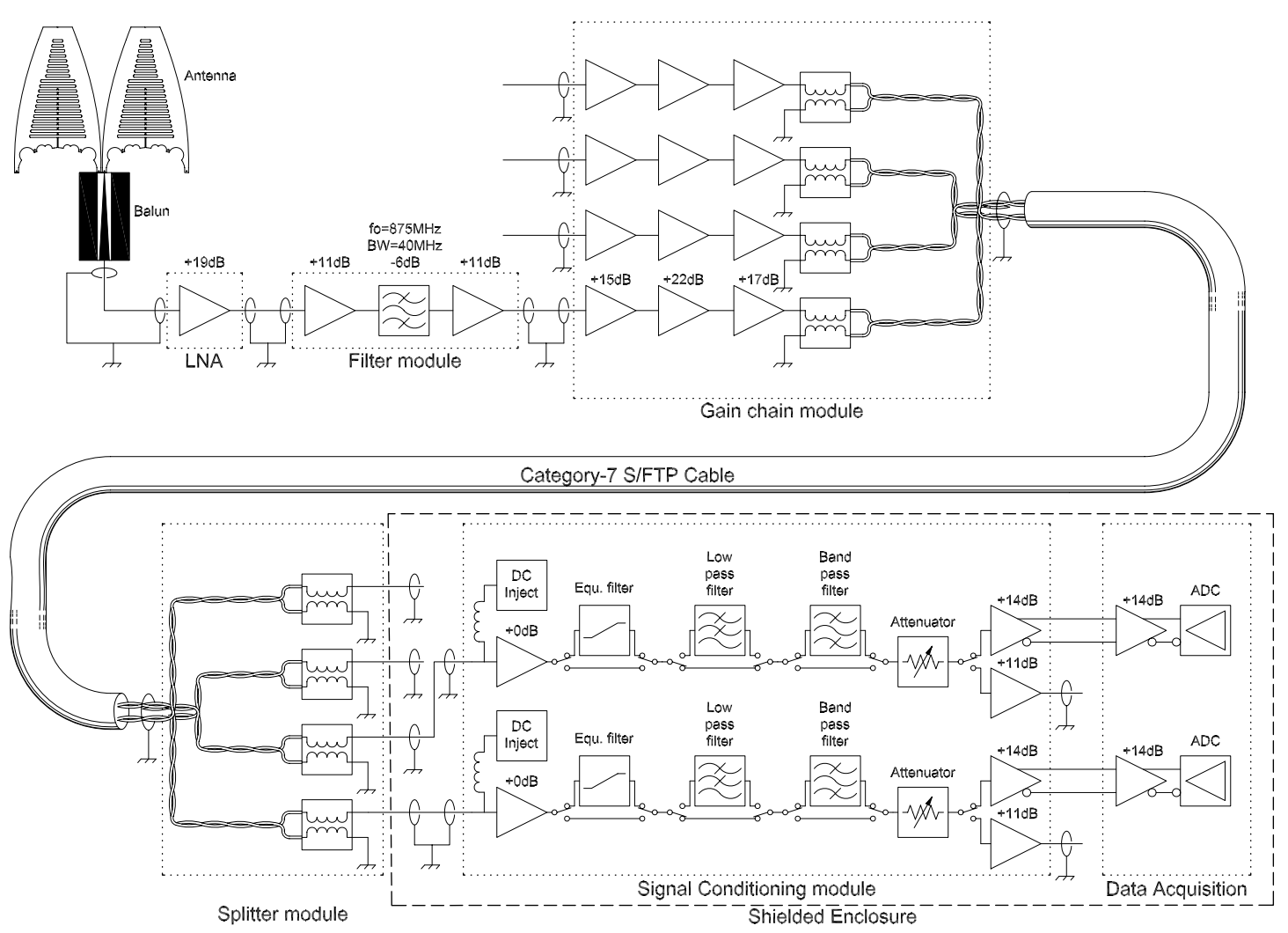

Fig. 1: System overview schematic. This figure shows one end to end chain of the analogue gain chain as used in 2PAD, from antenna to ADC.

At present 16 channels are populated with analogue back ends in a $4 \times 4 \times 1$ configuration. In the very near future this will be expanded to a $4 \times 4 \times 2$ array. The components used in $2 \mathrm{PAD}$ described in this paper were either Commercial-Off-The-Shelf modules, or discrete components on specially designed printed circuit boards.

\section{Transmissions}

Due to the requirement to deliver RF signals reasonably free of interference, it was necessary at an early stage in the project to survey the spectrum covered by the design. This was undertaken by use of an early prototype single row antenna array, and a portable spectrum analyser. Measurements were taken located on the then future site of 2PAD, by pointing the array in various lateral directions. From these measurements, it was possible to identify and exploit a reasonably wide $40 \mathrm{MHz}$ band centred on $875 \mathrm{MHz}$.

The spectrum from $300 \mathrm{MHz}$ to $1000 \mathrm{MHz}$ which $2 \mathrm{PAD}$ was generally designed to observe is richly endowed with television broadcast and Global System for Mobile (GSM) transmissions. As with any plane area in the UK, Jodrell Bank is in range of three major TV transmitter masts, with Winter Hill, (Fig. 2) in Lancashire being the most powerful. Analogue transmissions from this mast are $0.5 \mathrm{MW}$.

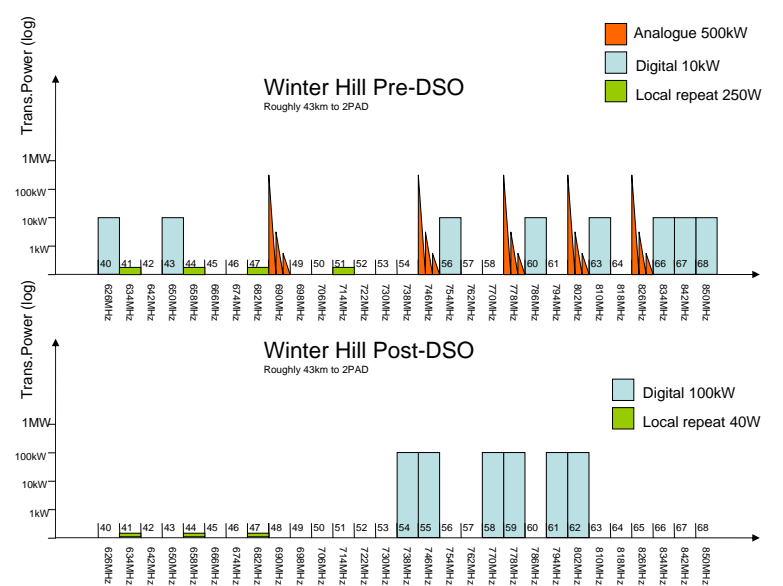

Fig. 2: Transmissions from Winter Hill mast. Source: www.ukfree.tv

In the UK the broadcast spectrum is in a state of change as existing analogue transmitters are switched off in the move to a national digital television broadcast coverage in a process known as Digital Switch Over (DSO). As a result of these changes digital broadcasts will be shifted in frequency and power increased by $10 \mathrm{~dB}$. However, the new digital transmis- 
sion power at $100 \mathrm{~kW}$ per channel will be small in comparison to the present analogue power.

The UK communications regulator, Ofcom, is supervising the Digital Switch Over. Of interest, the band between $790 \mathrm{MHz}$ and $854 \mathrm{MHz}$ are being cleared to make way for alternative commercial broadcasts (Ofcom 2009).

\section{Antenna}

Three antenna arrays have been built and fully characterised (Zhang \& Brown 2009). The array selected to be mounted on 2PAD in the short term is the Bunny Ear Combline Antenna array. This array has produced successful active array pattern scans in anechoic chamber tests and has met its design specification. In future a differential input low noise amplifier (LNA) will be used as the first amplifier in the analogue system. Such an LNA will be tailor made to match the output impedance characteristics of the antenna. However, until such an amplifier becomes available, a balanced to unbalanced transformer (balun) has been used instead. (Fig. 3)

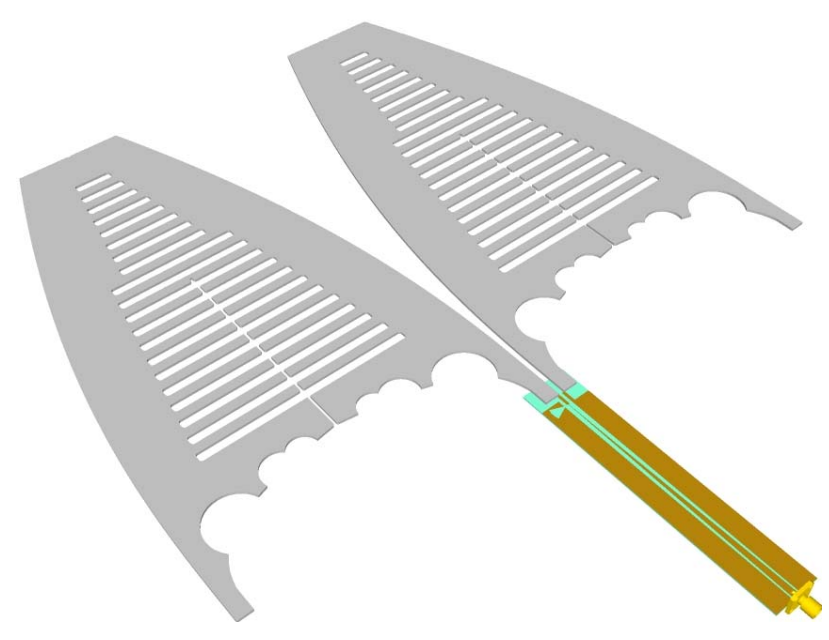

Fig. 3: BECA Antenna blade with CPW balun attached.

\section{Balun}

For the electromagnetic characteristic study of the antenna array, it was necessary to connect the feed terminals on the balanced antennas to an unbalanced coaxial cable. This transition requires not only a balanced-to-unbalanced transformation circuit, but also an impedance change due to the different impedance characteristics of the antenna and the cable. In the case of the BECA antenna the nominal impedance is $150 \Omega$,

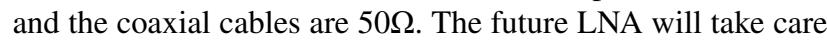
of both impedance matching and the mode conversion. In the short term, however, a balun has been fabricated on a high dielectric constant substrate, AD1000, provided by Arlon, a substrate manufacturer in the USA. This balun provides a transition between the coplanar waveguide (CPW) of the antenna and the coplanar stripline (CPS) required by the coaxial connector.
Any balun of this type is lossy. Measurements made using a test balun give a varying $\mathrm{S} 21$ between $0.11 \mathrm{~dB}$ at $500 \mathrm{MHz}$, and $0.5 \mathrm{~dB}$ at $1000 \mathrm{MHz}$. Use of a balun such as this defines the noise performance of the whole system, therefore is not a preferred solution for a sensitive telescope. However, the balun may be viewed as a place-holder for a future differential LNA.

One design issue was how to connect between the aluminium feed point on the antenna and the copper of the balun without causing a point of corrosion at the junction. To this end a $2 \mathrm{~mm}$ screw fixing was used with a corrugated beryllium washer between the two metals. This has proved to be a reliable solution for this short-term fixture.

\section{LNA}

The LNA used in this instance is a packaged module manufactured by Mini-Circuits ${ }^{\mathrm{TM}}$ Inc. This is the ZX60-33LN, which is a small un-cooled package with SMA and power connectors. This module provides nominally $20 \mathrm{~dB}$ of gain. The quoted noise figure of this device is $1.1 \mathrm{~dB}$. Since the noise performance of 2PAD is dominated by the lossy balun at the antenna, this LNA was accepted as satisfactory in this initial application.

One important feature of this amplifier is the dynamic performance. With an output $1 \mathrm{~dB}$ compression point of $+16.5 \mathrm{dBm}$ this unit is able to linearly amplify the broadband spectrum received by the antenna, including the powerful analogue TV transmissions, without introducing significant intermodulation products.

The DC supply to this LNA was set at $3.0 \mathrm{~V}$ by a linear regulator in the adjacent filter module. Connection to the input of the LNA was by SMA back-to-back adapter, and to the output by means of a $150 \mathrm{~mm}$ long conformable semi-rigid cable.

\section{Filter Module}

The small filter module is provided to exploit part of the spectrum relatively free of broadcast interference. It uses a Surface Acoustic Wave (SAW) band-pass filter manufactured by K R Electronics, Inc. The filter has a center frequency of $875 \mathrm{MHz}$, a bandwidth of $40 \mathrm{MHz}$ and out of band rejection of $>55 \mathrm{~dB}$. On the PCB either side of the filter, $11 \mathrm{~dB}$ of gain is provided by two monolithic amplifiers. Since the filter has a pass-band insertion loss of $6 \mathrm{~dB}$, the net gain is $16 \mathrm{~dB}$.

The power supply for this module is minimum $7.0 \mathrm{~V}$, maximum of $10 \mathrm{~V}$, which is fed from the Gain Chain Module, described in section 8. Linear regulators in the circuit feed the on-board amplifiers and the external LNA.

An alternative configuration for this module is in broadband attenuator mode, whereby a $20 \mathrm{~dB}$ attenuator is inserted in the path instead of the filter and gain. This is set by using the alternate two SMA connectors on the module. The use of this mode allows observation of a strong artificial source with wide bandwidth options. The broadband attenuator mode offers limited sensitivity compared to the amplified narrow band mode.

The PCB is soldered to the walls of its own shielded steel enclosure, which has fingered lids for electrical continuity (Fig. 4). 


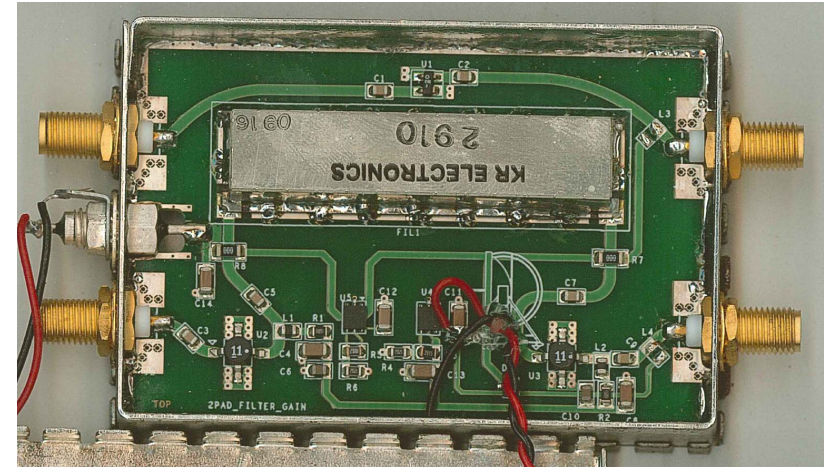

Fig. 4: Filter module with two monolithic gain blocks.

\section{Gain Chain Module}

Four RF signals are treated in one Gain Chain Module. Using three monolithic gain block amplifiers, $54 \mathrm{~dB}$ of gain is applied to each of the input signals. A transformer is then used to convert the single ended $50 \Omega$ signals to $100 \Omega$ differential, ready to be connected to the twisted pairs of the category-7 cable. The circuit uses linear regulators to power the on-board amplifiers. An unregulated DC supply is fed to the external filter module and the LNA. The PCB is housed in a rugged aluminium enclosure. This unit was designed and built by the University of Oxford.

Whilst the Gain Chain Module provides acceptable performance when the input RF power is carefully limited, the design is under review with the aim to improve input match, cross-talk performance, noise immunity and dynamic range.

\section{Category-7 Cable}

The cable system selected for analogue signal transfer on 2PAD has been the TERA ${ }^{\circledR} 1200 \mathrm{MHz}$ S/FTP cable from the Siemon Cable Company. This has four twisted pairs which are individually foil screened, with an overall braid screen. This cable is specified up to $1200 \mathrm{MHz}$. Targeted primarily at the $10 \mathrm{~Gb} / \mathrm{s}$ Ethernet market this cable has exceeded all performance requirements for 10GBASE-T (Savi 2004).

The reasons for selecting such a cable are as follows:

- Reduced cable area per channel.

- Reduced connector footprint at the bunker manifold.

- Reduced cost for analogue signal transport.

- Ability to use the same cable for the DC power supply to the front end.

On the first three points it has been found that CAT-7 cable does have merits when compared to the traditional coaxial cable. However, the spectral roll-off of the cable as the frequency increases does have an impact on the whole system. It requires a significant amount of extra gain in the system, leading to stability problems, and also the addition of an expensive equalisation filter. With a coaxial cable both these complexities could be avoided.

This cable and others have been extensively measured (Kibet et al. 2008) and proved to be the best performing ca-

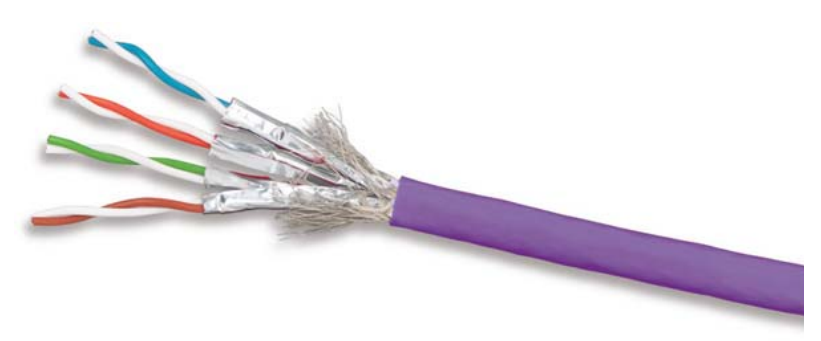

Fig. 5: TERA ${ }^{\circledR} 1200 \mathrm{MHz}$ cable (Source: Siemon Cable Company)

ble of its type on the market at the time, in terms of cross talk and insertion loss. Kibet measured a steady roll-off in S21 for the cable from $-9 \mathrm{~dB}$ at $500 \mathrm{MHz}$ to $-13 \mathrm{~dB}$ at $1000 \mathrm{MHz}$ on a 20 metre cable. The $\mathrm{S} 21$ slope is repeatable and shows a smooth roll-off to beyond $1.5 \mathrm{GHz}$.

This cable provides a convenient means of transferring DC power supply to the LNA and front end electronics. This is by means of bias- $T$ inductors placed at either end of the trans fer circuit. There is considerable power loss involved in this method, and this is an item for review in the continuing project.

Connection is made to the CAT-7 cable at either end by means of the Siemon Cable Company TERA connector? This connector provides excellent pair-to-pair shielding performance, so for the cable and connector Near End Cross Talk (NEXT) is kept within manufacturers specifications. NEXT is quoted by the manufacturer as typically $83 \mathrm{~dB}$ at $1000 \mathrm{MHz}$ for a $100 \mathrm{~m}$ length of this cable. The isolation specification for the analogue transport was $50 \mathrm{~dB}$ for a $20 \mathrm{~m}$ length, and therefore we may be confident that this cable will meet the design crosstalk requirements.

Kibet et al. have also measured the transformer extensively used in this system for translating between balanced and unbalanced signal forms. This is the ADTL2-18+ available from Mini-Circuits ${ }^{\mathrm{TM}}$ Inc, which based on measured S11 and S21 performance was found to work satisfactorily up to $1.5 \mathrm{GHz}$.

\section{Splitter}

A non-trivial challenge was raised when providing ingress of copper wires through the shielded walls of the bunker. There was legitimate concern that radiated or conducted interference would leak out of the bunker along with the cable.

An experiment with RF choke tubes fitted to the outside of the bunker (King 2007) demonstrated that with no cables inserted, the choke function was working effectively. King measured a shielding effectiveness of $92 \mathrm{~dB}$ at $1420 \mathrm{MHz}$. However, as soon as a wire was inserted through the tubes, the shielding effectiveness decreased to $48 \mathrm{~dB}$. The conclusion from this experiment was that the shield was breached since the wire and tube effectively form a coaxial cable. Attempts at using aluminium foil as a screen had little measurable effect on the shielding performance.

Following advice an effective solution was designed where the transformers were mounted onto PCBs inside a specially built galvanised steel enclosure (Fig. 6) The enclosure pro- 
trudes into the shielded bunker, and the single sided coaxial connectors are in full $360^{\circ}$ contact with the steel of the shielding interface. When attempting to produce a costed design, it is important not to forget the fabrication costs of shielded enclosures. This is an area for further research, since the cost of good shielding can quickly outstrip the cost of the electronics it encloses.

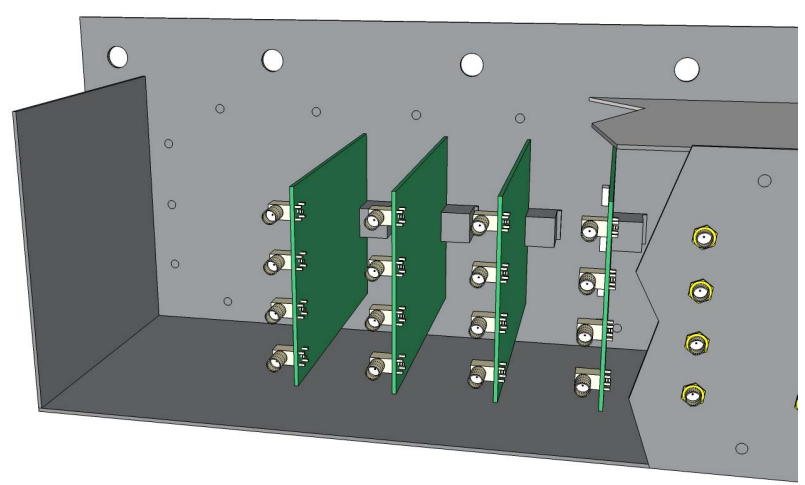

Fig. 6: Cut away sketch of Splitter Enclosure

Initial measurements (van der Merwe et al. 2009) have suggested that this design does not compromise the shielding effectiveness of the bunker. Signals injected onto the inside bunker wall at a power of $0 \mathrm{dBm}$ at $500 \mathrm{MHz}$ were not traceable from the outside using either a common mode current clamp or Log Periodic Dipole Antenna measured to a sensitivity of $-100 \mathrm{dBm}$. It is accepted this is a first-order test, and future measurements will cover greater bandwidths and will be more sensitive to take account of the large gains present in each receiver chain.

\section{Signal Conditioning Module}

The Signal Conditioning Module (SCM) (Fig. 7) provides the final treatment of each signal before digital conversion. This is a very flexible unit which allows various signal paths and conditions to be selected by remote control. RF filter components may be installed or removed and connectivity is by SMB connectors.

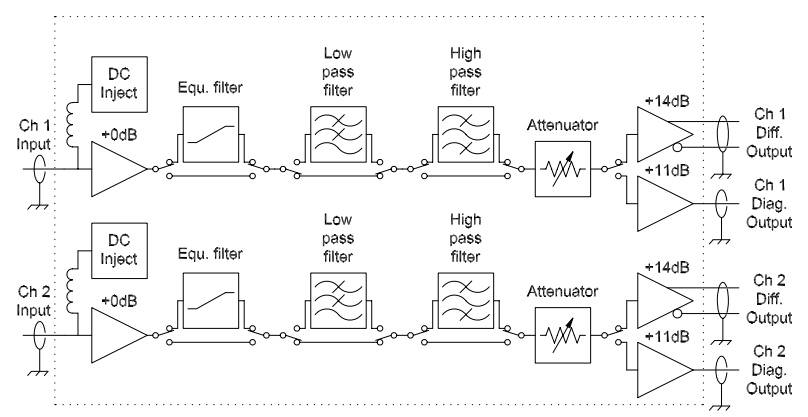

Fig. 7: Schematic description of Signal Conditioning Module
Firstly the spectral slope introduced by the CAT-7 cable is corrected in an equalisation filter. This simply provides an equivalent inverse slope, similar to a first order high pass filter.

Two filters are then optionally selected. These are to select one of two available Nyquist zones of the sampler. The ADC samples at a rate of $1 \mathrm{GS} / \mathrm{s}$, which sets the Nyquist frequency at $500 \mathrm{MHz}$. This means one may supply signals in two spectral bands:

a. $0 \mathrm{MHz}$ to $500 \mathrm{MHz}$,

b. $500 \mathrm{MHz}$ to $1000 \mathrm{MHz}$.

Any signal which appears in a non-selected zone will be aliased into the selected one. For this reason the filters have been specified to have strong out of band rejection at $>50 \mathrm{~dB}$.

The SCM has the facility to insert attenuation into individ ual channels. This was to allow for fine trimming of the voltage seen by the ADC. A five bit digital step attenuator may be used to insert between $0 \mathrm{~dB}$ and $32 \mathrm{~dB}$ in steps of $1 \mathrm{~dB}$.

A series of RF switches are used to route the signal path to each filter or to bypass it.

Signals pass from the SCM in differential mode in order to increase noise immunity.

A front panel connector was provided for diagnostic purposes which allows monitoring of the RF signal as seen by the ADC but in single ended mode. A monitoring amplifier is provided which may be selected by means of a toggle switch.

All settings on the SCM are remotely controlled by an Instrument Management Subsystem, a distributed serial interface under the control of a master control computer. This interface is linked to the SCM from the Data Acquisition Card. An alternative Translator Board provides crude manual control of the SCM. Two 16-bit words from the remote controllers are fed onto the $\mathrm{SCM}$ on an $\mathrm{I}^{2} \mathrm{C}$ bus. Data from the bus is distributed to $\mathrm{I}^{2} \mathrm{C}$ bus expanders which then directly drive each control section on the SCM.

This module was specified by the University of Manchester and designed and built by the University of Oxford.

\section{Future Development}

As part of the present PrepSKA project it is proposed to expand the $2 \mathrm{PAD}$ array from its present $4 \times 4 \times 2$ configuration to a $8 \times 8 \times 2$ array provided in the same antenna package. The delivery of a stable differential low noise amplifier is imminent, and should permit observation of some bright astronomical objects.

PrepSKA offers a good opportunity for a top down review of the implementation of the analogue system. Some discussions to be had are:

- power delivery method for the front end electronics

- where the digital conversion should be, at the antenna or in a bunker?

- the analogue signal transport medium to be used, if any.

Several research groups within SKADS and PrepSKA are making effective use of RF over fibre instead of copper cables for dishes. This technology should be formally reviewed for aperture arrays. However the use of any long analogue signal transport cable introduces losses and complexities, which could be reduced or eliminated by use of alternative topologies. 
The power budget for the Aperture Array in the Square Kilometre Array is extremely demanding. For example, the whole analogue system from antenna to the input of the ADC has a target power specification of only $200 \mathrm{~mW}$ (UK SKA Consortium 2008). In the present implementation on 2-PAD the focus was performance rather than reducing cost or power, and presently the power drawn is 6.2 Watts for each analogue chain (Price et al. 2010). Future work will begin to study means of compressing the analogue system into a few integrated circuits, which will help to reduce power drawn, cost and complexity. The amplifiers presently used in a prototype system such as 2PAD require a lot of power as a means of providing a large dynamic range. Future designs will therefore need to limit the dynamic range to the minimum required to adequately drive the ADC, with a view to reducing power demand.

\section{Conclusions}

The use of the CAT-7 cable was initially suggested as a means to reduce the physical connector footprint at the shielded enclosure, and to reduce cost. At first glance CAT-7 is superior to coaxial cables in both these respects. However, the loss introduced in the cable demands a complex analogue system with large amounts of gain. This can lead to problems with stability. Further, when used as a medium for DC power supply, the loss induced in the cable is significant.

The complexity of this prototype analogue system has been demanded as we have built up experience. However, as we move toward a SKA deliverable design we shall be finding ways to reduce cost and complexity by means of integration. One the most critical areas is the power required to drive the analogue section of the aperture array, and this must constrain the future design effort. Alternative topologies will be examined which will be aimed at a future large-scale roll out of a stable, cost effective and low noise aperture array.

RFI will be a feature of any earth-bound radio observatory, not least the Square Kilometre Array. The RFI environment is one of the key points in the ongoing site survey by the SKA Project Design Office. The potential for self pollution with radiated RFI through the use of digital electronics must be robustly countered with effective shielding built into the design from the outset.

The design and construction of 2PAD has been an excellent experience, which will contribute to future designs of aperture arrays. 2PAD is the first phased array of its type made by the UK radio astronomy community, and has potential not only for growth of engineering expertise, but also as a useful tool for astronomers learning to use this innovative type of telescope.

Acknowledgements. We would like to thank the following for their invaluable work in building, testing and measuring the components of the system: Richard Morrow, Richard Whitaker, John Edgley at Jodrell Bank, and Danny Price at Oxford Astrophysics. Thanks to Mina Panahi for the simulation of the analogue system. Thanks to Georgina Harris for her enthusiastic leadership. Thanks to the all the people who have generously given their time to review this paper.

\section{References}

van Ardenne, A. ASTRON, NL 2004. The European Aperture Array SKA Demonstrator. Electronic MultiBeam Radio Astronomy ConcEpt (EMBRACE)

Kibet, P.L., van der Merwe, P.S., Reader, H.C., Stellenbosch University, Siemon CAT7 Cables, Balun and Connector Measurements. Internal Technical Note: ITN 08b-08.

King, I.J., European EMC Products Ltd., 2007. Radio Frequency Shielded Test Report on Shielded Penetration Assembly. Report on study commissioned by the University of Manchester.

van der Merwe, P.S. \& Reader, H.C., 2009, Stellenbosch University, Notes on EMI investigation for 2-PAD.

Ofcom, 2009. Digital dividend: clearing the $800 \mathrm{MHz}$ band. www. ofcom. org.uk

UK SKA Consortium, 2008, Preparation for the SKA: PrepSKA.

Price, D., Schediwy, S., Ikin, T., Jones, M., "The 2 PAD Analogue System: Characterisation, Commissioning, and Implications for Calibration and Beamforming," SKA Memo \#122, 2010, available from http://www. skatelescope.org/pages/documents_3.htm

Savi, O, 2004. 10G Ethernet Over Structured Copper Cabling. www. siemon.com

Zhang, Y. \& Brown, A. 2009. Integrated Aperture Array Antenna Design for Radio Astronomy. Proceedings of SKADS Conference 2009 